\title{
POLITIQUES DE L'IMAGE : APPARITION DE L'HUMAIN ET RÉSISTANCE À PARTIR DE JUDITH BUTLER ET GEORGES DIDI-HUBERMAN
}

DANIELLA PRIETO ARRUBLA

\begin{abstract}
In this paper different philosophical approaches are re-examined in regard to the question how humanity appears in images. Firstly, based on Judith Butler's analysis of the photographic frame, the determination of the images by the relations of power is addressed. Secondly, in the context of DidiHuberman's comments on Pasolini and the fireflies figure as form of resistance, the possibility to resist against the enforced framing by taking otherness and its appearing into account is at issue. Lastly, referring to Hito Steyerl's theory of contemporary documentary art, I analyse the of ethical aspect of the posed question. Multiple examples of contemporary Colombian documentarism as well as one case of art in the context of the Jewish persecution during World War II are offered as an illustration of the ideas presented in this paper.
\end{abstract}

\section{Introduction}

Dans ce texte je me propose d'analyser, à partir de la reprise de plusieurs perspectives, la manière dont l'humain apparait dans l'image. En premier lieu, je me réfère au travail de Judith Butler sur le cadrage photographique établi dans un régime de pouvoir et non seulement en interprétant la réalité, mais déjà en l'instaurant. Cette perspective permet une première considération de l'image comme soumise à l'action du pouvoir, en tant que cadre strictement défini et contraint par des normes sociales et politiques. Ainsi émerge la question de la possibilité de résistance au cadrage fixe. En deuxième lieu, j’introduis la référence à Georges

https://doi.org/10.14712/24646504.2020.6

(C) 2020 The Author. This is an open-access article distributed under the terms of the 82 Creative Commons Attribution License (http://creativecommons.org/licenses/by/4.0). 
Didi-Huberman et à son travail La survivance des lucioles qui permet à travers la métaphore des lucioles, prise de Pier Paolo Pasolini, de considérer une forme de résistance au pouvoir, dans laquelle l'humain apparait. Pour ce faire, je me réfère dans un premier temps au travail de Didi-Huberman sur l'art documentaire comme exemple de résistance, puis dans un deuxième temps, je propose quelques exemples de documentaires colombiens afin de montrer la structure de l'apparition de l'image, d'abord comme imposition et manipulation du pouvoir, mais aussi comme un processus de résistance en réponse à cette imposition. De plus, j'approche brièvement la thématique de l'Holocauste en considérant le travail de l'artiste Friedl Dicker-Brandeis qui fait apparaître l'humain à travers les images dessinées par des enfants au ghetto de Terezin. Finalement, le travail de Hito Steyerel permet de poser explicitement la question de la tâche éthique du documentaire et de revendiquer ce lieu de résistance. Cette même revendication sera développée tout au long de ce travail en tant qu'expression de cette tâche éthique qui consisterait d'un côté à montrer le cadrage de l'image établi par le pouvoir étatique, et d'un autre côté à ouvrir ces espaces de l'apparition de l'humain.

Ainsi, la question fondamentale, tout au long de ce travail, n'est pas seulement comment l'humain apparait dans l'image, mais comment cette apparition peut se faire à la manière d'une résistance au pouvoir, même dans le cas limite de la disparition. Cette expérience nous permettra de constater le caractère obscur et clandestin de ces formes d'apparition qui sera visible à différents moments du texte, et d'observer une constellation de pensées pour réfléchir à l'image, à son rapport au pouvoir, et à la résistance comme sa tâche éthique.

\section{Judith Butler et la délimitation du cadrage photographique}

Dans le chapitre « La torture et l'éthique de la photographie : penser avec Susan Sontag ${ }^{1}$ de Ce qui fait une vie, Judith Butler présente une compréhension du cadrage photographique en tant que cadrage de la réalité même. Cela signifie une délimitation - faite de manière active - de ce qui apparait dans le cadre : l'établissement même de ce qui compte comme réel. Butler explique :

En fait, si la notion d'interprétation visuelle n'est pas vouée à devenir un oxymore, il semble important de reconnaitre que, en cadrant de la réalité, la photographie a déjà

1 Butler Judith, Ce qui fait une vie : Essai sur la violence, la guerre et le deuil, traduit de l'anglais par Joëlle Marelli, Paris, Zones, 2010, pp. 65-100. 
déterminé ce qui comptera à l'intérieur du cadre - et cet acte de délimitation est sans aucun doute un acte d'interprétation. ${ }^{2}$

Le cadre ne limite pas simplement l'image, il la structure, et c'est pour cette raison que dans le cas de la photographie de guerre, la question doit se poser non seulement sur le contenu de l'image, sur ce qui se montre, mais aussi sur la manière dont elle le montre. La manière, dit Butler, "n'organise pas seulement l'image, mais aussi notre perception et notre pensée ${ }^{3}$. Ici Butler s'accorde avec Susan Sontag sur le fait qu'il s'agisse de structurer activement notre appréhension cognitive de la guerre; d'une régulation des modalités visuelles de participation à la guerre, des manières dont les citoyens disposent pour voir ce qui se passe, et en conséquence, réagir ou non dans le champ politique. Il s'agit en effet d'une régulation de la disposition même du sujet, de l'affect, et de la sensibilité éthique. La relation entre établissement de la réalité et le cadre photographique peut être observée de manière privilégiée dans la pratique du journalisme embarqué qui s'est imposé avec l'invasion de l'Irak, lors de laquelle les journalistes ont dû montrer la guerre en suivant les paramètres et les règles établies par le Ministre de la Défense des États-Unis de telle manière que le cadre même et la perspective qui s'y trouve constituent déjà l'interprétation de la guerre imposée par l'État.

En se référant à son livre précédant Vie précaire, Butler insiste sur la reconnaissance de normes sociales et politiques qui sont déjà déterminées par la question de savoir où et quand une vie peut être ou non sujette au deuil. ${ }^{4}$ Les normes déterminent ce qui est représentable en tant que tel à travers la délimitation d'un cadre visuel et l'exclusion de certaines parties. L'image produite de cette façon apparaît comme perceptible conformément aux normes déterminées par le cadre, et précisément par son accord à ces normes il détermine aussi ce qui est perceptible à son intérieur. Ainsi Butler affirme : "L'image censée livrer la réalité le soustrait en fait à la perception. $»^{5}$

Butler réfléchit soigneusement autour de la relation entre photographie et atrocité. La photographie sert de preuves des crimes de guerres; elle établit la vérité de cette accusation, elle confirme que l'atrocité a effectivement eu lieu. Mais si la photographie constitue la preuve, elle constitue aussi l'accusation. En outre, Butler considère cela comme une exposition de la façon dont les médias élaborent

\footnotetext{
Ibid., p. 69.

Ibid., p. 73.

Ibid., p. 65, p. 75.

Ibid., p. 76.
} 
ces arguments, c'est-à-dire que la photographie, même lorsqu'elle semble transparente, a été cadrée à une certaine fin. Comme elle l'écrit :

Si nous supposons que cette fin est l'interprétation, alors il apparait que la photographie interprète encore la réalité qu'elle enregistre, et cette double fonction est préservée alors même que la photographie est offerte comme "preuve » d'une autre interprétation présentée sous une forme écrite ou verbale. Après tout, la photographie ne se contente pas de renvoyer à des actes atroces, mais élabore et confirme ces actes pour ceux qui les appelleraient ainsi. ${ }^{6}$

Ainsi même, la photographie déjà comme cadrage est postérieurement recadrée dans certains discours. Butler propose une réflexion autour des photographies du cas de torture à Abou Grhaïb pendant la Guerre d'Irak, qu' elle considère comme " une occurrence implicite de journalisme embarqué " ${ }^{7}$. Elle explique : "L'angle de l'appareil, le cadre, la pose des sujets, tout suggère que ceux qui prenaient ces photos étaient activement engagés dans la perspective de la guerre. ${ }^{8}$ Ainsi, dans ces photographies, il y a non seulement une représentation de l'autrui, mais également de l'autrui vers celui contre qui se fait la guerre, considéré comme l'ennemi. La torture même présuppose et au même temps réinstaure la non-humanité de l'autre, l'être moins qu'humain du sujet torturé9. Elle se fait dans l'ignorance des conventions légales au moyen de la présupposition que ce sujet n'a pas le statut d'humain, et donc des droits déterminés.

\section{Didi-Huberman et la résistance au cadrage imposé : laisser apparître I'humain}

Devant ce scénario, on pourrait se demander comment est-il possible de résister et de s'opposer à ce cadrage imposé. Et surtout dans quelle mesure peut-on articuler l'image et l'éthique dans le monde contemporain ? En se référant à Guy Debord, il est possible de décrire notre monde contemporain en tant que société du spectacle, comme un monde saturé et aveuglé par « la lumière crue, cruelle, féroce de la marchandise " sans laisser aucune place pour d'autres formes d'expérience, pour d'autres formes de représentation et apparition. ${ }^{10}$ Face à ce type

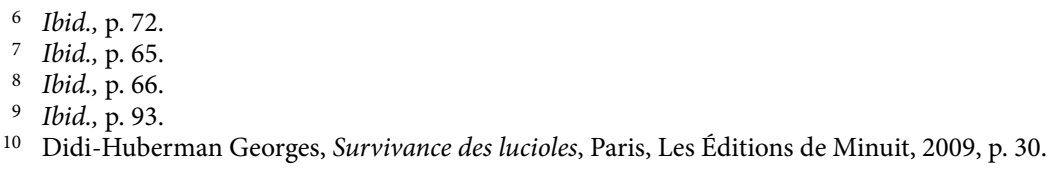


de questions, Georges Didi-Huberman entreprend ses réflexions dans Survivance des lucioles. ${ }^{11}$ Il utilise la figure des lucioles, ces petites lumières d'une splendeur discrète, qui s'opposeraient à la grande lumière du fascisme. Les lucioles feraient de la résistance en cherchant à se défendre et à s'échapper de la situation oppressive au moyen d'échange de petits signaux de lumière discrets. Didi-Huberman mentionne dans ce texte Pier Paolo Pasolini comme sujet central, qui, à travers plusieurs moments de sa vie, avait écrit et décrit la lumière du fascisme et de ces lucioles. Pour Pasolini, c'est une erreur de considérer que le fascisme italien a été vaincu après les années 1930 ou 1940. En réalité, il considère que le fascisme a continué de différentes manières dans la société italienne et qu'il y a aussi une émergence des nouvelles formes fascistes. Le fascisme est défini pour lui à travers sa relation à la disparition de l'humain, une forme d'assimilation totale qui pourrait être appelée " génocide » ou " génocide culturel » ${ }^{12}$. Ainsi, tout comme dans la description de Butler, chez Didi-Huberman la figure de l'humain est centrale. Mais Didi-Huberman, en se référant à Pasolini, met également l'accent sur le caractère communal de l'humain, sur la figure du peuple en tant que puissance de résistance. La résistance n'appartient pas aux intellectuels, qui, comme le dit Pasolini, « même les plus avancés et les plus critiques, ne s'étaient aperçus que 'les lucioles étaient en train de disparaître” » ${ }^{13}$. Pasolini trouve dans le populaire, "l'énergie révolutionnaire propre aux misérables, aux déclassés du jeu politique courant $»^{14}$.

Dans son texte "L'article des lucioles ", Pasolini décrit le fascisme italien comme une dissimulation qui se base sur le vide de pouvoir, qui règne en réalité en Italie, puisque le pouvoir réel ne se trouve pas chez les dirigeants politiques, de manière que cet exercice du pouvoir soit seulement formel :

Je dis «formellement » parce que, je le répète, dans la réalité, les notables démocrates-chrétiens recouvrent, par leurs manœuvres d'automates et leurs sourires, le vide. Le pouvoir réel avance sans eux : il ne leur reste entre les mains que ces appareils inutiles ne livrant plus d'eux que la réalité de leurs funestes complets vestons. ${ }^{15}$

Ce pouvoir qui se trouve au-delà est un pouvoir de l'industrie et du consumérisme, qui traverse la totalité de la vie humaine, les corps, les âmes, et même jusqu'au désir. C'est ce que Pasolini appellerait aussi un "néo-capitalisme

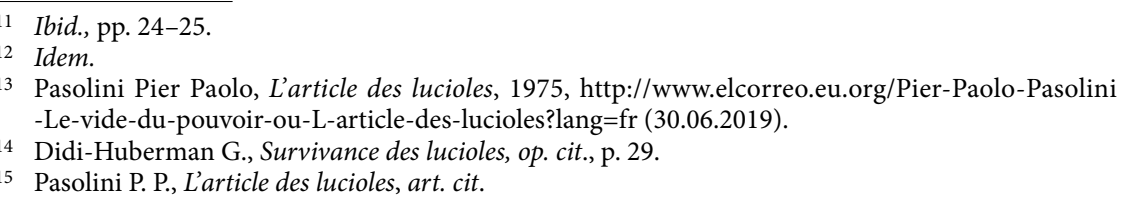


télévisuel », dont l'image et l'art même ont été assimilés par le pouvoir totalisant. ${ }^{16}$ Dès lors, Didi-Huberman affirmerait que l'imagination est politique, que sous la façon d'imaginer gît une façon de faire de la politique, c'est-à-dire une politique de l'image.

La question serait, en conséquence, comment faire apparaitre les peuples et l'humain même dans l'image. Didi-Huberman considère "L'article des lucioles " de Pasolini de 1975, comme le signe d'un désespoir et d'un désenchantement politique. Pasolini affirme que les lucioles ont disparu et que ce peuple italien, que lui-même avait aimé, a souffert lui aussi d'une défiguration sans retour. La résistance populaire ne lui semble plus possible. Cependant, Didi-Huberman cherche à maintenir cette possibilité ouverte, en demandant si les petites fulgurations résistantes ont réellement disparu, si ce n'est pas notre tâche de les chercher et de les observer, de « se donner les moyens de voir apparaitre les lucioles dans l'espace du présent $»^{17}$.

Pour montrer un cas d'images clandestines dans lesquelles l'humain apparait, Didi-Huberman propose le documentaire Border (2004) de Laura Waddington. Comme dans le cas des photographies de torture analysées par Judith Butler, il s'agit ici d'images dans lesquelles le visage humain n'est pas complètement visible. Dans l'expérience clandestine et momentanée de traverser le frontière, les visages humains restent dissimulés par le caractère illégal même de l'action. Butler avait, quant à elle, trouvé dans cette caractéristique la trace même de l'humain, de sa souffrance et de son oblitération. ${ }^{18}$ Pour elle l'expérience de la douleur est liée à notre animalité. C'est précisément dans l'animalité qu'il peut y avoir de la souffrance. ${ }^{19}$ Ici, dans le documentaire de Waddington, les figures humaines qui cherchent à traverser la frontière apparaissent comme des irruptions fulgurantes dans l'obscurité de la nuit, parmi les ténèbres de la désolation et de la solitude. Cette apparition est toujours intermittente et en train de disparaitre, puisque les migrants ne cessent d'être en mouvement. Ils sont aussi des survivants, ce qui reste d'une communauté en voie de disparition. Waddington raconte : "Il y avait des enfants aussi, personne ne les attendait, ils n'avaient nulle part où aller. J'avais un ami proche, un gars de Kaboul, il était là avec son jeune frère, ils avaient perdu ses parents près de la frontière au Pakistan. $»^{20}$ Les histoires comme celles-ci

\footnotetext{
Didi-Huberman G., Survivance des lucioles, op. cit., p. 30.

Ibid., p. 59.

Butler J., Ce qui fait une vie : Essai sur la violence, la guerre et le deuil, op. cit., p. 94.

Ibid., p. 76.

Waddington Laura, Border, 2004, 7 min. $58 \mathrm{sec}$ - 9 min. $17 \mathrm{sec}$., la traduction est la mienne. https:// www.youtube.com/watch? $\mathrm{v}=0 \mathrm{X} 42 \mathrm{CxOomqQ}$ (28.11.2019).
} 
abondent. Waddington décrit aussi les recherches policières et les rencontres avec les migrants. Comme dans les lettres de Pasolini, les projecteurs utilisés pour les recherches, ici les lampes torches des policiers (« les faisceaux des torches, les aboiements des chiens $»^{21}$ ), sont aussi de grandes lumières du pouvoir et de ses mécanismes de contrôle. Sur les photographies de Abou Grhaïb, Butler considère que bien que la photographie ne définisse pas l'humain, l'humanité quant à elle est bel et bien en jeu dans la photographie. ${ }^{22}$ De manière semblable, Susan Sontag avait remarqué la nécessité, la tâche, de laisser les images nous hanter. ${ }^{23} \mathrm{C}^{\prime}$ est aussi par un cri fantomatique, venu lui aussi nous hanter, que l'on entend s'affronter la police française et les migrants dans une des scènes du documentaire de Waddington. Dans ce même documentaire, l'une des questions s'oriente vers l'image que nous laissons apparaître de l'autre, et comment, dans cette image, on aperçoit la preuve d'un traitement de l'autre avec moins d'humanité, un autre qui est en quelque sorte pourchassé et victime de la brutalité.

Waddington raconte que Didi-Huberman lui a envoyé son livre sur les lucioles. Ensuite, elle a écrit un court texte sur ce même thème à la demande d'une revue lui dédiant une édition. Dans ce texte elle écrit :

Quand j'ai lu Survivances des Lucioles dans ce café internet de Jordanie, j'aurais voulu pouvoir trouver Abdullah. J'aurais aimé lui offrir ce livre qui parle de Forough Farrokhzad, ce livre avec sa croyance dans les liens silencieux de la transmission et du hasard - des fils presque invisibles, mais pas tout à fait. [...] Cette nuit-là à Sangatte, alors qu' Abdullah, assis par terre sous la pluie, parlait des poèmes de Forough Farrokhzad, je sais que les lucioles étaient là. ${ }^{24}$

\section{Démonter le cadrage : laisser les lucioles apparaître}

La question est toujours fondamentalement sur l'humain, sur la possibilité de faire apparaitre l'humain dans l'image, ce que Didi-Huberman appelle, en suivant

21 Ibid., 12 min. 5 sec. -12 min. $12 \mathrm{sec}$., traduction prise des sous-titres de YouTube, https://www .youtube.com/watch?v=0X42CxOomqQ_(28.11.2019).

22 Butler J., Ce qui fait..., op. cit., p. 79.

23 Ibid., p. 97.

24 Waddington L., Abdullah et les lucioles - á la lecture de Survivance des Lucioles de Georges Didi Huberman, traduit de l'anglais par Charlène Dinhut et Aude Tincelin. Citée du site web de Laura Waddington, URL: https://www.laurawaddington.com/articles/51/abdullah-et-les-lucioles-a-la -lecture-de-survivance-des-lucioles-de-georges-didi-huberman (15.06.2019). 
Arendt, «parcelles d'humanité » 25 et qui pourrait, en retournant à la réflexion de Susan Sontag, susciter une réponse éthique. La question pour Sontag se concentre sur la possibilité de réagir politiquement, de réévaluer notre position envers la guerre. Il s'agit en tout cas de voir l'autre ainsi que sa douleur. Cet autre est ce qui constitue une culture. La question de l'interculturalité peut être exotisée de manière à la voir comme un récit de multiples cultures. Mais l'autre n'apparait pas toujours comme un autre qui vient d'une autre culture. L'autre est parfois tout proche de nous. Il peut être incarné par ces figures féminisées, de migrants, ou d'homosexuels. L'autre peut être aussi ce qui souffre de l'incapacité corporelle. Penser la rencontre entre les humains demande bien plus que de penser le mélange de cultures. L'interculturalité demande d'envisager l'autre dans sa singularité : l'autre, proche de nous, est souvent invisibilisé. Sous les lumières du pouvoir, du spectacle, de notre monde de consumérisme, apparaissent toujours les mêmes images, les mêmes visages. Mais quels sont les visages que cette politique de l'image efface et que ces projecteurs féroces n'illuminent jamais? Et comment se fait-il que, lorsqu' ils apparaissent, leur apparition soit cadrée, déterminée par le pouvoir même? Dans notre société contemporaine l'autre apparait souvent par l'image humanitariste. Cette image, qui sert aussi comme justification des actions colonialistes et interventionnistes sur l'autre, s'utilise également afin d'obtenir des bénéfices économiques. Cette manière de vendre la misère, le chagrin de l'autre, a été thématisée par les cinéastes colombiens Luis Ospina et Carlos Mayolo au moyen du concept de pornomiseria ou "porno-misère ", en tant que forme de misérabilisme à des fins mercantiles tel qu' exposé dans leur court métrage "Agarrando pueblo» (Les Vampires de la misère), puis dans un petit texte intitulé « Qu' est-ce que la Porno-Misère ? » ${ }^{26}$. En partant du cas du cinéma colombien, ils décrivent une "porno-misère » généralisable à l'ensemble des médias :

Le cinéma colombien indépendant a eu deux origines. Une première qui visait à interpréter ou à analyser la réalité et une deuxième qui découvrait, à l'intérieur même de cette réalité, des éléments anthropologiques et culturels afin de la transformer. Au début des années 1970, avec la loi de soutien au cinéma, un certain type de documentaire est apparu, qui copiait superficiellement les réalisations et les méthodes des cinémas indépendants jusqu'à les dénaturer. Ainsi, la misère est devenue un thème choquant, une marchandise facile à vendre, à l'étranger en particulier, où la misère est la contrepartie de l'opulence des consommateurs. Si la misère avait servi d'élément de dénonciation

25 Didi-Huberman G., Survivances des lucioles, op. cit., p. 131.

26 Ospina Luis et Mayolo Carlos, Qu'est-ce que la Porno-Misère, traduit de l'espagnol par Olivier Hadouchi et Joaquín Manzi, 2014, https://rsomnambules.wordpress.com/2014/07/06/quest -ce-que-la-porno-misere-par-luis-ospina-et-carlos-mayolo/ (23.06.2019). 
et d'analyse pour les cinémas indépendants, l'appât du gain n'offrait pas une méthode susceptible de découvrir de nouvelles prémisses pour analyser la pauvreté. Bien au contraire, ce nouveau cinéma indépendant et misérabiliste a créé des schémas démagogiques jusqu'à devenir un genre que l'on pourrait appeler la porno-misère. ${ }^{27}$

On peut interpréter la posture de Judith Butler comme suggérant une manière de s'opposer à la politique de l'image, à ses règles de cadrage, en montrant le cadrage même, en réfléchissant à son montage et en observant ce qui se cache derrière lui. Oscar Campo, un vidéaste colombien, propose un exercice de réflexion sur l'apparition des images de l'autre dans le contexte du conflit interne colombien, ce qui pourrait être, me semble-t-il, en accord à ce type de tâche. Dans son documentaire ou essai visuel "Cuerpos frágiles " (Corps fragiles), le vidéaste présente un montage ou une accumulation d'images audiovisuelles tirées de journaux télévisuels et de matériel d'internet au sujet du conflit armé colombien ${ }^{28}$. Dans ces images capturées par les journaux télévisuels dans le cadre de ce conflit interne, on retrouve une manière de faire apparaître les corps, de parler d'eux, qui semble obscène et incorrecte à Campo. Ces images montrent, à de nombreuses reprises, les corps de guérillas, de combattants terroristes abattus sur le champ de bataille ; ce sont des corps fragmentés et mutilés dont la mort doit signifier le bonheur d'autres personnes. À partir de ce point, Campo expose la relation entre médias et militaires, entre forces armées et publicité, comme une relation dans laquelle le destin de certains corps est déjà déterminé d'avance par le pouvoir médiatique. De la même manière, le pouvoir médiatique est cadré par le gouvernement, par son discours de sécurité démocratique, de défense de la société, et d'une certaine haine qui est nécessaire pour soutenir la normalité. La mort de certains corps, leur torture et leur déchirement sont non seulement justifiés, mais sont aussi postérieurement exposés et exhibés par les médias comme une victoire. Il y a aussi d'autres corps, des corps de militaires, d'autres agents du conflit qui sont aussi présents dans les médias et qui apparaissent au moyen d'un usage manipulateur à des fins politiques ou publicitaires. Cette dynamique du spectacle des médias dans laquelle s'exhibent les corps l'un après l'autre, chaque événement effaçant le précédent, n'appartient pas uniquement à la politique colombienne, mais aussi à une politique de l'image présente au niveau global, et est une dynamique que Campo retrouve également dans le discours de " guerre contre le terrorisme» de Bush dans lequel les violences sur certains corps ne sont pas seulement justifiés, mais voulus. Un discours politique et médiatique où l'autre est relégué à un moindre statut, où l'autre

27 Idem.

28 Campo Oscar, Cuerpos frágiles, 2010, https://vimeo.com/52173241 (25.06.2019). 
n'importe pas, et pour cette raison son corps peut être exposé dans son déchirement, dans sa défiguration et sa mutilation, dans son anéantissement, en tant que cet autre corps, cible de la violence, l'ennemi, l'objectif militaire. Ces autres ne sont pas seulement les objectifs militaires, mais aussi ceux qui sont autour de lui. Dans son essai visuel, Campo montre les images de la vidéo Collateral Murder (2007) partagée par Chealsea Manning et publiée par Julian Assange (2010), qui, comme nous le savons, a été prise par la caméra embarquée d'un hélicoptère militaire dans la guerre d'Irak, et dans laquelle une partie des blessés étaient de la population civile, des journalistes et des enfants, dont la mort et les blessures sont assumées comme quelque chose de léger et de divertissant, encore une fois comme la mort dont Campo parle au début de son essai, une mort de quelques individus, pour laquelle il est acceptable de se réjouir ou de ne pas s'en préoccuper.

En retournant à Didi-Huberman, on trouve un autre exemple qui traite de la possibilité de résistance, qui est le cas des rêves collectés par Charlotte Berardt pendant la guerre en Allemagne, et qui servent comme reflet d'une société dominée par le fascisme, et de la crainte vécue par les citoyens dans ce contexte. A partir de la réflexion de Didi-Huberman au sujet des lucioles, je propose de considérer à la manière de la communauté de lucioles la communauté des enfants avec laquelle l'artiste Friedl Dicker-Brandeis avait travaillé dans le ghetto de Theresienstadt (Terezín) dans le contexte du génocide juif. L’artiste essayait d'utiliser l'art comme une certaine forme de résistance thérapeutique envers l'expérience traumatique de la guerre telle que vécue par les enfants, alors qu' elle occupait un poste d'enseignante en arts. Les enfants faisaient des dessins autour de différents thèmes proposés par Dicker-Brandeis dans lesquelles ils montrent leur vie dans l'enfermement, leur peur et leurs souvenirs de la vie avant d'être déplacés au ghetto. Ces dessins sont précisément ceux des lucioles en train de disparaître. Les enfants, comme des lucioles, ont été des survivants le temps d'un court instant, après que la guerre eut commencé et jusqu'à leur transportation au camp de concentration d'Auschwitz, où la plupart d'entre eux sont morts. À partir du travail de Butler il est possible de distinguer les images que le pouvoir instaure d'autrui, en délimitation du cadrage et d'une perspective déterminée. Maintenant, avec Didi-Huberman, il est également possible de considérer une autre catégorie d'image, celle produite par les lucioles survivantes, ou par leur exposition. Cette deuxième catégorie est envisagée comme une résistance et une subversion de la première. Il ne s'agit plus de la représentation que le pouvoir fait d'un groupe humain, mais plutôt la trace qu'il crée, comment ces humains apparaissent ou sont laissés apparaitre.

Ces travaux des enfants de Terezín sont exposés à la Synagogue Pinkas en République tchèque, sous la direction du Musée Juif de Prague. De plus, certains sont 
aussi publiés dans un livre étonnamment intitulé « Fireflies in the Dark (Lucioles dans l'obscurité), dans lequel l'écrivaine Susan Goldman Rubin raconte l'histoire du travail de Dicker-Brandeis avec les enfants. Le titre du livre de Goldman Rubin semble avoir été tiré de la pièce de théâtre également intitulée "Lucioles ", un conte de fées tchèque, que les enfants jouaient sous la direction de Dicker-Brandeis. Lors de ce processus les enfants auraient aussi fait des dessins de costumes ainsi que des extraits de la pièce, dans lesquels l'image des lucioles nous est donnée à voir.

\section{Hito Steryerl : Le travail documentaire et le dire vrai}

L'artiste allemande Hito Steryerl réfléchit au livre de Didi-Huberman Images malgré tout dans son essai «Le documentarisme en tant que politique de la vérité » ${ }^{29}$, dans lequel elle aborde, en référence à Foucault, la question de la relation entre la vérité et les rapports de pouvoir, dans ce cas-ci centrée sur les formes documentaires. Steryerl raconte que dans ce travail Didi-Huberman réfléchit sur les quatre photographies qui survécurent d'Auschwitz, et il les définit comme des moments de vérité. ${ }^{30}$ Elle précise :

Cette formulation signifie néanmoins deux choses : d'une part, ces photos participent sans aucun doute à la vérité, tout comme les images dialectiques de Benjamin. D'autre part, il serait, selon Didi-Huberman, inadmissible d'exiger de ces photos « toute la réalité ».31

Ces photographies étaient prises, comme l'explique Steyerl, par des prisonnières à Auschwitz dans l'idée de les rassembler comme preuves des atrocités qui eurent lieu dans le camp de concentration. Elles ont été prises dans la clandestinité, l'obscurité, la peur. L'inintelligibilité, le fait qu'elles ne soient pas complètement claires, font partie intégrante des images mêmes. L'un problème que posent ces images est précisément leur lien à l'histoire. Comment les utiliser, les montrer en tant que document historique ? Pour Steyerl - en suivant à Didi-Huberman - l'un de plus gros problèmes de ces photographies est que, en vertu de leur propre obscurité, elles ont été manipulées par des historiens et des muséologues pour les faire apparaitre plus plausibles ou plus propres, parfois au moyen « d'une

29 Steyerl Hito, Le documentarisme en tant que politique de la vérité, traduit par Yasemin Vaudable, 2003, https://eipcp.net/transversal/1003/steyerl2/fr.html (30.06.2019).

30

Idem.

1 Idem. 
contextualisation précise ou de 'l'inscription' de telles images ${ }^{32}$. En revanche, elle clarifie :

Ce qui est ainsi supprimé de l'image lors du cadrage - un extrait filmé de travers, le cadre sombre de la chambre à gaz, depuis laquelle deux de ces photos ont été prises, les parties floues et les parties brouillées - sont justement les aspects dans lesquels le moment de danger, tel que l'entend Benjamin, s'est nettement gravé, le moment d'une production en péril d'un intervalle de temps minuscule, qui perce le contrôle national-socialiste sur toute production d'image dans un endroit clairement délimité. ${ }^{33}$

Cette question nous renvoie vers Susan Sontag qui remarquait que les photographies ne peuvent pas être interprétées sans l'accompagnement de légendes et d'analyses écrites, et qu'en plus les photographies en tant que telles ne pourraient pas changer notre évaluation politique puisqu'elles n'offriraient pas de compréhension en raison de leur manque de cohérence narrative. ${ }^{34}$ Dans ce contexte, Butler avait répondu que les images n'ont pas besoin d'interprétation, qu'elles sont elles-mêmes déjà une interprétation de la réalité. Comme nous l'avons dit, l'image se présente dans une sphère sociale déterminée qui, pour Butler, fait aussi partie de la scène même de la photographie, elle-même sujet d'interprétation. Ainsi, Butler avait défini une double interprétation au cœur de l'image : d'un côté, l'image en tant que cadrage interprétatif de la réalité, et de l'autre, son interprétation postérieure, également cadrée. Dans les images d'Auschwitz, encore une fois, les images sont vues de manières différentes selon leur lieu de présentation. Il y a eu - comme le décrit Butler à propos de Abou Grhaïb - une manière établie par le pouvoir étatique de représentation visuelle : une politique de l'image. Dans ce cas, Steyerl explique :

Auschwitz était un territoire qui, certes, disposait d'un atelier de photographie mais qui ne pouvait en aucun cas être photographié par des personnes non-autorisées. C'est ainsi que des milliers de photos « officielles » d'Auschwitz furent prises, sur lesquelles l'on ne pouvait rien, absolument rien voir de l'extermination de masse qui y avait lieu. ${ }^{35}$

On distingue ainsi, dans ce cas, une opposition entre l'image cadrée par le pouvoir, et les images-lucioles prises par les survivants. À la fin de son texte, Steyerl remarque deux questions à développer. L'une est la question de l'éthique

\footnotetext{
Idem.

Idem.

Butler J., Ce qui fait..., op. cit., p. 68.

Idem.
} 
en relation au documentaire, et l'autre est une question qui concerne l'opposition, ce que nous avons appelé ici la résistance. Les deux questions sont articulées. Elle écrit : " Le caractère 'urgent' du documentaire réside dans le dilemme éthique consistant à devoir témoigner des événements, qui, en tant que tels, ne peuvent pas être communiqués mais contiennent nécessairement des éléments de vérité aussi bien que d'obscurité. » ${ }^{36}$ Selon elle, le documentaire possède dans son essence même une tâche éthique qui consiste à rendre possible d'autres visibilités, d'autres formes d'apparition que celles déterminées par le pouvoir. Elle conclue :

Il n'existe guère de visibilité, qui ne soit pas imprégnée - si bien que nous pouvons presque dire que ce que nous voyons est toujours déjà prévu par des rapports de pouvoir. D'autre part, le doute formulé à l'égard de ces visibilités persiste avec une opiniâtreté, elle-même capable de constituer une propre forme de pouvoir. ${ }^{37}$

En revenant au documentaire d'Oscar Campo au sujet de la représentation du corps dans le média politique, on peut reconsidérer son travail comme une pratique de cette politique de la vérité et de la tâche éthique du documentaire. On retrouve, dans le travail de Campo, une réflexion sur le rapport direct entre politique et média et le cadrage d'un discours sur le corps et sur autrui.

Le regard de l'image devrait se poser aussi sur l'autre, cet autre qui est marginalisé, ordinaire, qui est presque toujours exclu de la lumière des grands projecteurs et de la définition canonique du beau. Cependant, l'image ne peut pas être misérabiliste, elle doit résister à la représentation de l'autre en tant que moins qu'un, en tant qu'objet de pitié. Pour illustrer ce regard sur l'autre en évitant le misérabilisme, on peut regarder la pièce documentaire Andar con tacto (Marcher avec tact, 2002) de Juan Carlos Arias et Dixon Quitián, dans laquelle on suit une personne aveugle, dans sa vie quotidienne, qui apparait dans le cadre photographique telle qu' elle est et sans intervention pour nous émouvoir. ${ }^{38}$ Cependant, l'élimination du misérabilisme ne signifie pas que les images ne nous émeuvent pas, comme nous l'avons déjà mentionné en référence à Butler, Didi-Huberman et Steyerl, puisque l'art documentaire peut en réalité, et il le fait, susciter une réponse éthique, il peut nous montrer la douleur d'autrui et susciter de surcroît une réponse dans le champ politique.

\footnotetext{
Idem.

Idem.

Arias Juan Carlos et Quitián Dixon, Andar con tacto, 2002, https://vimeo.com/295909892 (Marcher avec tact) (30.06.2019).
} 


\section{Conclusion}

Au long de ce travail, nous avons considéré différentes pensées autour de l'image. De Butler à Steyerl les éléments centraux restent toujours les mêmes : le pouvoir et l'humain. Cependant, chacun de ces penseurs nous donne une manière différente de traiter la question, et souvent ils se complètent entre eux. La perspective de Didi-Huberman permet ainsi d'élargir les formes de résistance au cadrage imposé, tel que défini par Butler. Didi-Huberman, à travers Pasolini et l'image des lucioles, permet de penser l'humain, sa constante lutte, sa disparition, mais aussi spécialement sa réapparition, sa survivance.

Butler, qui reste dans un dialogue constant avec Susan Sontag, explicite les difficultés de penser la résistance à une image qui est déjà interprétation de la réalité et qui est elle-même en constante interprétation par les médias et par le pouvoir. Ces auteurs nous ont donné la possibilité et les éléments pour réfléchir autour d'exemples concrets d'art, comme ceux de Campo, Waddington, Friedl Dicker-Brandeis, qui s'approchent plus ou moins de l'un ou l'autre de ces penseurs, mais qui partagent un esprit critique et confrontent le pouvoir établi, l'apparition toujours des mêmes figures et la politique dominant de l'image. Ils montrent la possibilité d'apparaître le cadrage, de le démonter, de s'y opposer, d'ouvrir de nouvelles formes d'apparaître, dont aussi figures différents peuvent devenir visibles. Ces travaux ne sont pas simplement un recours nostalgique ou un espoir faux. Ils sont plutôt le résultat d'une tâche éthique qui concerne l'art en général et l'art documentaire spécialement.

Il reste toujours à examiner la façon avec laquelle le pouvoir montre l'autre, les visages qu'il laisse apparaître et ceux qu'il efface ; il reste à réfléchir sur la manière de s'opposer à cette imposition. Bien que les structures fondamentales restent en place, les temps changent, et aussi les technologies. Pour cette raison, malgré la reconnaissance de cette tâche éthique de l'art, de la question du comment laisser apparaître l'humain dans le cadre photographique ou dans l'image, il est nécessaire de se poser également la question concernant le mécanisme de fonctionnement de nos technologies, de comment s'opposer à la forme même dans laquelle l'appareil photographique détermine déjà ce qui apparait dans le cadre.

Hito Steyerl montre cette problématique de manière splendide dans le chapitre «Proxy Politics: Signal and Noise» de son livre Duty Free Art: Art in the Age of Planetary Civil War, dans lequel elle explique le mode de fonctionnement actuel des appareils photographiques des téléphones mobiles, leur mécanisme, ou 
ce qu'elle nomme « paradigme » de la photographie computationnelle ${ }^{39}$, qui fonctionne en utilisant les images prises avant l'image actuelle et les images de nos réseaux informatiques, pour surmonter la grande quantité de bruit dans l'image et ainsi produire une image suffisamment claire. «Le résultat peut être une image de quelque chose que n'a jamais existé, mais que l'algorithme pense que vous pourriez vouloir voir. Ce type de photographie est spéculatif et relationnel. C'est un pari avec des probabilités qui hasarde sur l'inertie. ${ }^{40}$ L'appareil photographique détermine donc déjà ce qui est considéré en tant que bruit dans l'image et ce qui est considéré en tant signal. Le problème est que, de cette façon, certains corps sont encore une fois exclus d'être considéré en tant que signaux et sont considérés immédiatement et automatiquement en tant que bruit ; cela se produit au moyen même de l'appareil photographique et non au moyen d'un sujet et de ses actions qui déterminent le cadre. La délimitation est donc plus grande que simplement l'orientation de l'appareil photographique dans une direction ou l'autre. Il faudrait donc d'abord se demander comment nos appareils, nos technologies, sont déjà immergés aussi dans un certain discours transmis par le pouvoir et comment ce discours détermine la façon même de fonctionner de l'appareil. Le problème central est ici, comme l'affirme Steyerl, « que cela rend plus difficile de voir des choses imprévus. Il augmente la quantité de bruit exactement comme il va augmenter la quantité d'interprétations au hasard. ${ }^{41}$ Les considérations de Steyerl montrent la nécessité d'actualiser la pensée autour de l'image, de reconnaitre les défis caractéristiques de notre contemporanéité et de nous situer dans les fractures que ce temps produit dans la subjectivité et l'affectivité, de reconnaitre la spécificité des façons de production des images aujourd'hui, qui se trouvent davantage traversée par la technologie et le pouvoir que jamais. En outre, ces appareils photographiques sont aussi ceux qui sont embarqués au cœur des systèmes de surveillance et de combats, qui déterminent par exemple l'objectif militaire vers lequel se dirige un missile ou une autre arme de guerre. Le dispositif militaire reçoit un signal qui détermine l'objectif, et décide qu'il est possible de tirer sur telle ou telle forme de vie et de l'anéantir. Steyerl montre aussi - comme on a déjà vu dans le cas de la vidéo de Collateral Murder, entre autre - comment certaines vies sont considérées dans la guerre contemporaine comme auxiliaires, ou non importantes, dont on

9 Steyerl H., Duty Free Art: Art in the Age of Planetary Civil War, London, Verso Books, 2017, p. 57.

$40 \quad$ Idem. La traduction est mienne. Original: "The result might be a picture of something that never ever existed, but that the algorithm thinks you might like to see. This type of photography is speculative and relational. It is a gamble with probabilities that bets on inertia."

41 Idem. La traduction est mienne. Original: "It makes seeing unforeseen things more difficult. It will increase the amount of noise just as it will increase the amount of random interpretation." 
peut disposer sans être inquiété. Dans le chapitre «Sea of Data», elle prend le cas d'une femme qui a perdu la vue après un bombardement sur sa maison alors qu'elle regardait la télévision. Et elle se demande : "Quel type de signaux a été extrait à partir de quel type de bruit pour suggérer que al-Nasasra était une cible légitime? Quels visages appariaient dans quels écrans, et pourquoi ? Ou pour le dire d'autre façon : Qui est signaux, et qui es jetable comme bruit ? » ${ }^{42}$ Ce serait l'une des questions que la pensée sur l'image devrait se poser de nos jours, qui nous permettrait de repenser la question de l'humanité, de la médiation, et de la résistance lors des défis propres à notre présent historique.

Daniella Prieto Arrubla est étudiante au master en philosophie dans le programme Master Erasmus Mundus Europhilosophie (Université Catholique de Louvain, Univerzita Karlova v Praze, Bergische Universität Wuppertal). Elle a étudié la philosophie à l'Universidad Javeriana en Colombie.

E-mail : daniellaprietoarrubla@gmail.com

42 Steyerl H., Duty Free Art: Art in the Age of Planetary Civil War, op. cit., p. 86. La traduction est mienne. Original: "What kind of 'signal' was extracted from what kind of 'noise' to suggest that al-Nasasra was a legitimate target? Which faces appear on which screens, and why? Or to put it differently: Who is 'signal', and who disposable 'noise'?" 